\title{
NEW VALUES FOR THE INFRARED ABSORPTION COEFFICIENT OF ATMOSPHERIC WATER VAPOR
}

\author{
By Walter M. Elsasser
}

[California Institute of Technology, A pril 1938]

Some time ago the writer examined those features of atmospheric radiation which ensue from the fact that the water vapor spectrum is a line spectrum and not a continuous spectrum (1). At that time it was not possible to give a quantitative redetermination of the absorption, since our spectroscopic knowledge of the far infrared region was not sufficiently advanced. In the meantime, the work of Randall, Dennison, Ginsburg, and Weber (2) has furnished us with a complete analysis of the far infrared line spectrum of water vapor covering the whole spectrum beyond a wave length of $18 \mu$. These authors have been able for the first time to resolve the spectrum completely into its individual lines; the wave lengths of the lines have been measured and their intensities, which are difficult to measure directly, have been determined by calculations based upon the quantum theory of the water molecule. On the basis of these important results, in combination with the rest of our knowledge of the water spectrum, it has now become possible to obtain improved numerical values for the absorption coefficient of atmospheric water vapor and its dependence upon pressure and temperature. Our calculations apply not only to the part of the spectrum covered in reference (2), but to the whole range of the infrared spectrum where the thermal radiation of the atmosphere and ground is appreciable. We might notice here that the necessity for taking the line structure of the water spectrum into account, in dealing with problems of atmospheric radiation, has been pointed out several years ago by $\mathrm{F}$. Albrecht (3).

\section{LINE ABSORPTION}

The spectrum beyond $20 \mu$ is covered by numerous and narrow spectral lines which are tabulated in (2). The form and properties of the spectral lines have been discussed in (1). If the total intensity of a spectral line is designated by $i_{0}$, then the intensity distribution of the line (i. e. the dependence of the absorption coefficient on frequency) is given by the formula

$$
i(\nu)=\frac{i_{0}}{\pi} \cdot \frac{\alpha}{\left(\nu-\nu_{0}\right)^{2}+\alpha^{2}} .
$$

Here $\nu_{0}$ is the frequency of the line center and $\alpha$ is a constant which has the dimension of a frequency. In spectroscopic practice it is customary to use reciprocal wave lengths $\left(\mathrm{cm}^{-1}\right)$ as a measure of the frequency scale; in order to obtain true frequencies $\left(\mathrm{sec}^{-1}\right)$, it is only necessary to multiply them by the velocity of light, $c=3.10^{10}$ $\mathrm{cm} \mathrm{sec}^{-1}$. We shall adopt this scale throughout.

The constant $\alpha$ is a measure of the width of the line; it can be assumed to be about the same for all lines in the spectrum; however, as explained in (1), it is proportional to the air pressure and inversely proportional to the square root of absolute temperature:

$$
\alpha=\alpha_{0} \frac{p}{p_{0}} \frac{\sqrt{T_{0}}}{\sqrt{T}}
$$

where $p_{0}$ and $\mathrm{T}_{0}$ are standard pressure and temperature. In (1), an estimate of the magnitude of $\alpha_{0}$ has been attempted. In the meantime this quantity has been measured by Cornell (4) for atmospheric absorption in the bands at $0.9 \mu$ and at $1.1 \mu$ and values of $0.29 \mathrm{~cm}^{-1}$ and $0.20 \mathrm{~cm}^{-1}$, respectively, have been found. These values are very much larger than the one estimated in (1); they are, however, in agreement with certain other experiences of band spectroscopy. We have no direct information as yet about the width of the far infrared lines; from general principles of atomic theory, it can, however, be inferred that the line width there should not differ appreciably irom that of the near infrared lines. A standard value of

$$
\alpha_{0}=0.25 \mathrm{~cm}^{-1}
$$

has, therefore, been adopted throughout in our following calculations. It must be emphasized that this value is by no means free from experimental uncertainties and may possibly be somewhat modified if more precise measurements of this type become available.

We proceed now to calculate the amount of radiation absorbed by spectral lines. If the width of the lines is

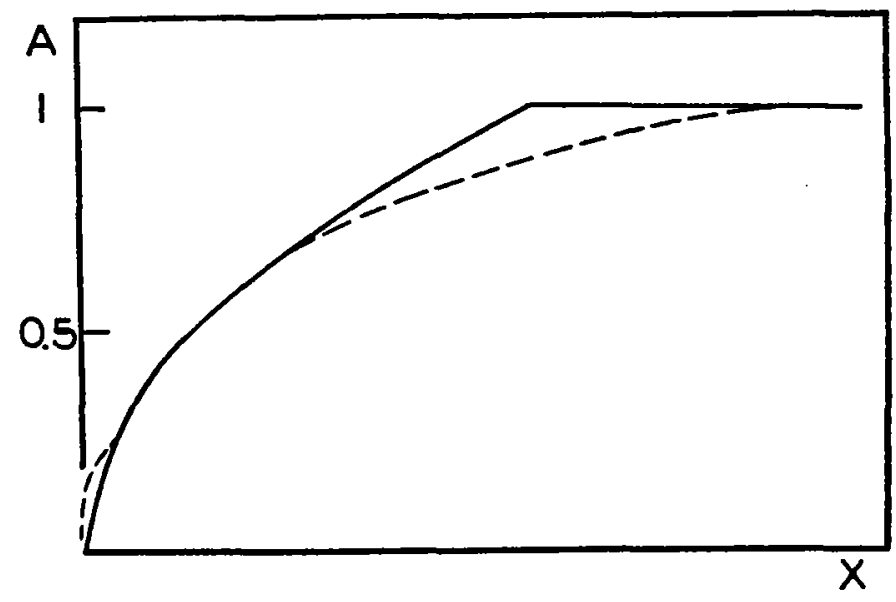

Figure 1.

small compared to the average distance between successive lines, the main part of the absorption takes place at a considerable distance from the line center and, as explained in (1), the formula (1) for the intensity distribution can then be replaced by the approximate expression

$$
i(\nu)=\frac{i_{0} \alpha}{\pi\left(\nu-\nu_{0}\right)^{2}}
$$

$i(\nu)$ means physically the amount of radiation absorbed in an infinitesimal layer of water vapor. In a layer of finite optical thickness $x$, say, the radiation will be weakened according to the law $I=I_{0} e^{-i x}$. We define now the fractional absorption in a layer of finite thickness as

$$
A=\frac{I_{0}-I}{I_{0}}
$$

and more generally as the fraction of the primary radiation absorbed in the layer. We can now readily compute the fractional absorption due to the presence of a single spectral line. Consider a spectral interval $\Delta \nu$ so large 
that the absorption due to the line is only appreciable inside it and is negligible outside it; on the other hand, it might be small enough that inside it the light intensity can be considered a constant independent of frequency. We define $A$, then, as

$$
A=\frac{\mathcal{S}\left(I_{0}-I\right) d \nu}{\int I_{0} d \nu}=\frac{1}{\Delta \nu} \mathcal{S}\left(1-e^{-i(\nu) x}\right) d \nu .
$$

The limits of the interval $\Delta \nu$ are also the limits of the integration; but on account of the assumptions made, we can, without appreciable error, replace these limits by $-\infty$ and $+\infty$. We have, then, by (4):

$$
A=\frac{1}{\Delta \nu} \int_{-\infty}^{+\infty}\left(1-e^{-\frac{i_{0 \alpha x}}{\pi\left(\nu-\nu_{0}\right)^{2}}}\right) d \nu=\frac{2 \sqrt{i_{0} \alpha x}}{\Delta \nu} .
$$

If the interval contains several spectral lines which are so far apart from one another that each can be assumed to

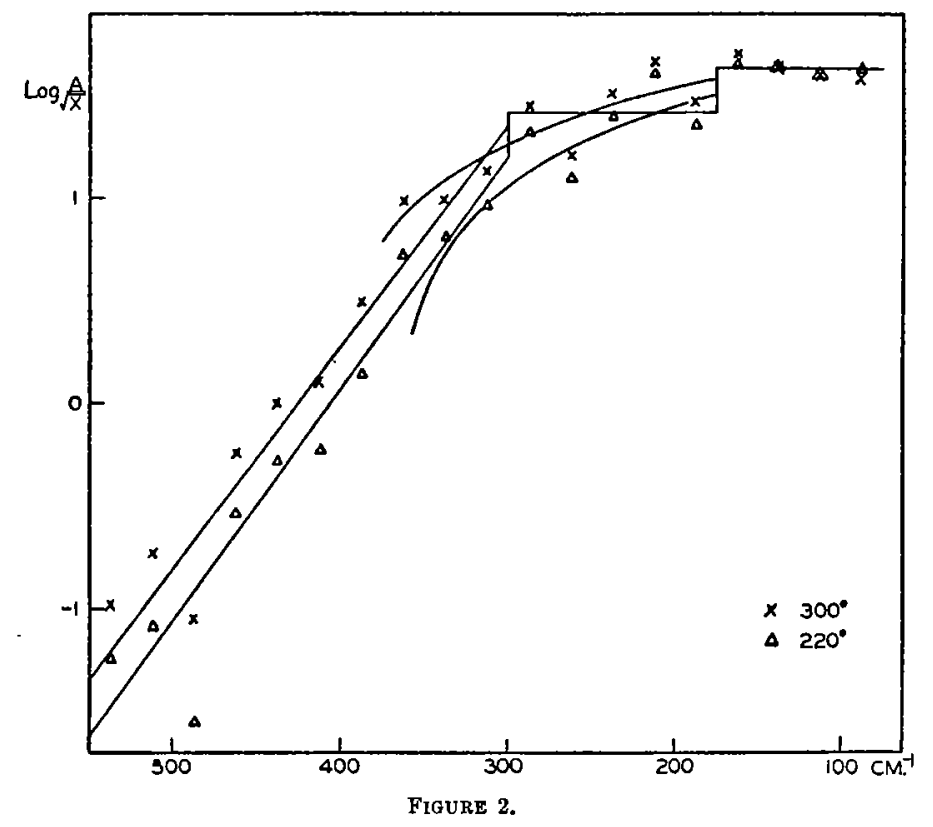

absorb independently from the others, we will, for the fractional absorption, have a sum of terms having all the form of the right-hand side of (5). Introducing the expression (2) for $\alpha$, we have finally

$$
A=\frac{2 \sqrt{\alpha_{0}}}{\Delta \nu} \sum \sqrt{i_{0}} \sqrt{\frac{p}{p_{0}}} \sqrt{\frac{T_{0}}{T}} \sqrt{x}
$$

where the summation extends over all lines in the interval $\Delta \nu$. The absorption is, therefore, proportional to the square root of the optical mass $x$ (we shall measure $x$ in $\mathrm{cm}$. of precipitable water); it is proportional to the square root of the air pressure and inversely proportional to the fourth root of the absolute temperature. The latter statement is, of course, only true if the pressure and temperature can be assumed constant throughout the layer; if they are variable, more complex formulae will result. We see here that the absorption of a line spectrum is fundamentally different from the simple exponential absorption which is valid for a continuous spectrum. C. Pekeris (5) has first pointed out the importance of the square root law for atmospheric radiation and has also shown that it is experimentally fulfilled in the near infrared atmospheric water bands measured by Fowle.

By definition, the quantity $A$ varies between zero and unity. The square root law is an approximation which becomes invalid for very small thicknesses, where the substitution of the approximate formula (4) in place of (1) is no longer legitimate; and also for very large thicknesses where neighboring lines overlap and where, therefore, the absorption is no longer the sum of the contributions of the individual lines. In fact, for large values of $x$, the absorption must asymptotically approach unity (fig. 1). It seems, however, a fairly good approximation to assume that the square root law holds up to the value $A=1$, while for still larger thicknesses $A=1$ remains valid. This schematic course of the fractional absorption is represented by the solid line in figure 1, while the broken line gives an idea of the true value of the absorption.

We come now to the numerical determination of the absorption. For reasons which will appear later, we shall divide the whole spectrum into three parts, the first ranging from the short wave end to about $10 \mu\left(1000 \mathrm{~cm}^{-1}\right)$, the second from about $10 \mu$ to about $20 \mu\left(500 \mathrm{~cm}^{-1}\right)$, and the third from there towards the long wave end. We start our investigation at the long wave side.

\section{ABSORPTION BEYOND $20 \mu$}

All the lines and their intensities are tabulated in (2). The absorption is given by formula (6). The dependence on temperature which appears in (6) gives, however, not the whole variation of the absorption with temperature, since, as explained in (1) in more detail, the line intensities $i_{0}$ also depend somewhat upon temperature in a more complex manner. In order to calculate the absorption, the spectrum was divided into intervals of $25 \mathrm{~cm}^{-1}$ width each, and the expression $\sum \sqrt{i_{0}}$ was calculated for a number of different temperatures. In figure 2 the results are shown for two temperatures; the points are located in the middle of the respective intervals, and the crosses correspond to $300^{\circ}$ abs. The ordinates are proportional to $\log A$ for a fixed thickness. The details of these calculations, which are too long to be reproduced here, are given in (6). The irregularities of the points in figure 2 are due to the irregular distribution of the water lines, which is not completely averaged out in the summation over the frequency intervals, although each interval contains a considerable number of lines. Now in order to have formulae which are practically applicable, the results have to be further smoothed out. The intensity of the absorption decreases in the mean exponentially for $\nu>300 \mathrm{~cm}^{-1}$. By a leastsquare method, a straight line was fitted to the ten points which lie beyond $300 \mathrm{~cm}^{-1}$ in the diagram. This was done for temperatures $200^{\circ}, 220^{\circ} \ldots$ up to $320^{\circ}$. The two lines for $220^{\circ}$ and $300^{\circ}$ are indicated in the figure. The results can be summed up in the following formula:

$$
\left\{\begin{array}{l}
A=10^{a_{\nu}+b} \sqrt{\frac{p}{p_{0}}} \sqrt{x} \\
a=-0.0108+8.4 \times 10^{-6} t \\
b=4.56 \\
\quad \text { for } \nu>300 \mathrm{~cm}^{-1}
\end{array}\right.
$$

where $t$ is the temperature in centigrade, $t=\mathrm{T}-273$, and the optical thickness $x$ is measured in grams of precipitable water.

For longer waves the absorption varies less rapidly and is also less dependent upon temperature. Since here no 
very great precision is required, the black body emission being already rather small, we have simplified the formulae by assuming the absorption to be constant in each of two intervals and to be independent of temperature:

$$
\begin{cases}A=26 \sqrt{\frac{p}{p_{0}}} \sqrt{x} & \left(300 \mathrm{~cm}^{-1}>\nu>175 \mathrm{~cm}^{-1}\right) \\ A=42 \sqrt{\frac{p}{p_{0}}} \sqrt{x} & \left(175 \mathrm{~cm}^{-1}>\nu>75 \mathrm{~cm}^{-1}\right)\end{cases}
$$

The measurements in (2) do not go beyond $75 \mathrm{~cm}^{-1}$; the absorption for still longer waves is, however, roughly known and may sufficiently well be approximated by extrapolating the second formula (8) down to $25 \mathrm{~cm}^{-1}$ and putting $\mathrm{A}=0$ between 0 and $25 \mathrm{~cm}^{-1}$.

For some purposes, an alternative approximation is more convenient. As seen from figure 2, the absorption is rather small beyond $375 \mathrm{~cm}^{-1}$ (the scale is logarithmic!) and is negligible in certain cases. The absorption intensities can fairly well be approximated by making $A$ (not its logarithm) a linear function of $\nu$ between 175 and 375 $\mathrm{cm}^{-1}$

$$
\left\{\begin{array}{l}
A=65.4+0.085 t-0.164 \nu \\
\text { for } 375 \mathrm{~cm}^{-1}>\nu>175 \mathrm{~cm}^{-1}
\end{array}\right.
$$

This function, plotted for $220^{\circ}$ and $300^{\circ}$, appears in the figure as a curved line. For $\nu$ smaller than $175 \mathrm{~cm}^{-1}$, the second formula (8) may be used.

\section{ABSORPTION ON THE SHORT WAVE SIDE}

We consider now the absorption caused by the strong water band which extends from $5 \mu$ to $8 \mu$. Since this absorption covers a relatively smaller part than the one treated above, we can do with a less detailed analysis. The general conditions are, however, the same as before, the absorption being caused by numerous narrow lines, and the square root formula (6) applies. We have only to determine the constant which enters in (6) and its dependence on frequency. In order to do this, we have combined various measurements available in the literature.

It follows from the precise spectroscopic analysis that the band center is located at $1595 \mathrm{~cm}^{-1}$, or, in sufficient approximation for our purposes, at $1600 \mathrm{~cm}^{-1}$. The band is very approximately symmetrical with respect to the center. We can use the curve given by Hettner (7) to determine the relative intensity of the absorption as function of the frequency. A sufficiently good approximation is obtained if $A$ decreases linearly at both sides of the center. The slope of these lines was determined by plotting the average absorption as given by Hettner on a frequency scale. The straight lines so determined cut the axis $A=0$ at $1250 \mathrm{~cm}^{-1}$ and $1950 \mathrm{~cm}^{-1}$, respectively. However, as pointed out in (1), measurements made in steam should not be used to determine the absolute amount of the absorption under atmospheric conditions. For the latter purpose, the measurements of Fowle (8) made in the atmosphere are suitable. We shall first try to check the square root law in Fowle's measurements. For this purpose the values of $A$ for various spectral intervals, as given by Fowle (9), are plotted with $\sqrt{x}$ as abscissa. This has been done in figure 3 . It is seen that up to a certain thickness, namely $0.1 \mathrm{~cm}$. of precipitable water, the measured points fall well on a straight line. Then the curve bends around and the following points lie on another straight line which does not go through the origin. It is difficult to determine the cause of this rather surprising phenomenon. One may presume that it is connected with the fact that the two sets of points are obtained experimentally by two different arrangements. The lower points correspond to a path of the light which remains inside the spectroscope itself, while for the measurement of the higher points an additional path of $117 m$ in air outside the spectroscope was required. Since there is a considerable amount of stray light which has to be taken into account as a correction, it was thought that this might be connected with the discontinuity which appears in figure 3 . For the following computations, only the lower section of the curves was used. The three intervals $5-6 \mu, 6-7 \mu$, and $7-8 \mu$, can be used for the comparison. If the relative values of the absorption, as resulting from the relative slope of the

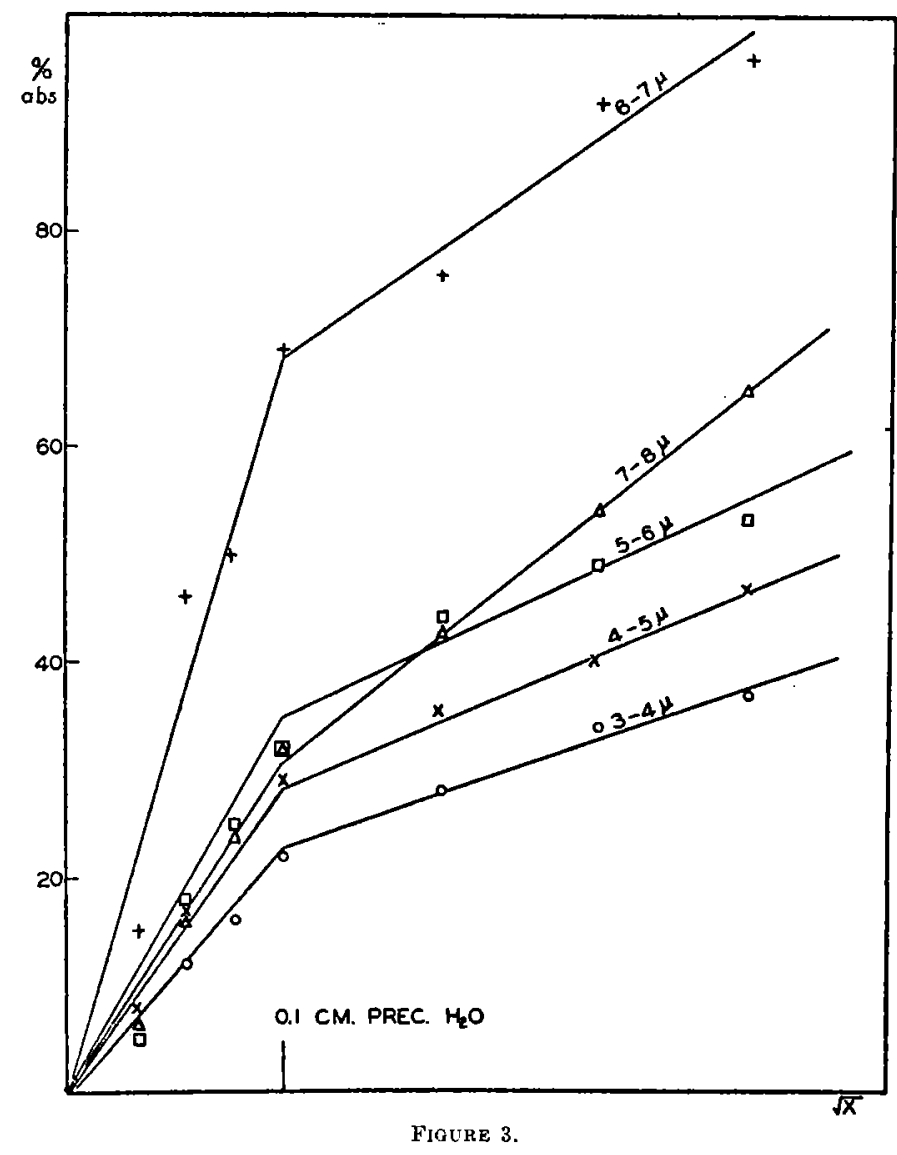

lines, are compared with Hettner's relative values, a certain discrepancy appears. One may either average the three intervals for the comparison, or pick out one

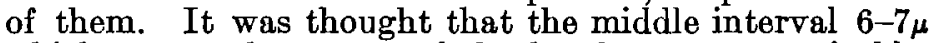
which covers the center of the band was most suitable, since the absorption extends over a rather large range (up to 70 percent), and the experimental error involved should, therefore, be considerably smaller in this case than in the other cases. We have, for the interval 6-7 $\mu$, an absorption $\mathrm{A}=2.2 \sqrt{x}$. Comparing this with the relative variation of the absorption with frequency which results from Hettner's data, the following formulae were obtained:

$$
\left\{\begin{array}{l}
A=3.7\left(1-\frac{\nu-1600}{350}\right) \sqrt{\frac{p}{p_{0}}} \sqrt{x} \\
\text { for } 1950 \mathrm{~cm}^{-1}>\nu>1600 \mathrm{~cm}^{-1} \\
A=3.7\left(1-\frac{1600-\nu}{350}\right) \sqrt{\frac{p}{p_{0}}} \sqrt{x} \\
\text { for } 1600 \mathrm{~cm}^{-1}>\nu>1250 \mathrm{~cm}^{-1}
\end{array}\right.
$$


The variation of this absorption with temperature should be so small that it has been thought appropriate to neglect it completely.

\section{ABSORPTION IN THE INTERMEDIATE REGION}

The spectroscopic analysis carried out with great resolving power shows that there are only a few extremely weak water lines in the region between $8 \mu$ and $25 \mu$. Nevertheless, an absorption of the atmosphere must be assumed in order to account for the measured amount of radiation coming in from the sky. We shall now investigate the way in which this radiation originates $(10)$.

It results from formula (1) or formula (4) that each line extends in principle to an infinite distance from its center. Absorption can, therefore, be caused by rather distant lines, provided only these lines are strong enough. This is the case for the absorption between $8 \mu$ and $25 \mu$. The intense lines which cause this absorption are located in the very far infrared, namely beyond $30 \mu$. The intensities of these lines are known (2). Now from (4) we see immediately that the absorption coefficient $i$, produced at the frequency $\nu$ by these lines, is equal to

$$
i(\nu)=\frac{\alpha}{\pi} \sum \frac{i_{0}}{\left(\nu-\nu_{0}\right)^{2}}
$$

and from (2):

$$
i(\nu)=\frac{\alpha_{0}}{\pi} \frac{p}{p_{0}} \frac{\sqrt{T_{0}}}{\sqrt{\bar{T}}} \sum \frac{i_{0}}{\left(\nu-\nu_{0}\right)^{2}}
$$

The sum extends over all the lines in the far infrared, and for each line its center frequency $\nu_{0}$ has to be substituted. The numerical calculations can readily be carried out; the results for $T=220^{\circ}$ and $T=300^{\circ}$ are given in the following table. For convenience, the variation of $i$ with the inverse square root of the temperature as expressed in (11) has already been taken into account. If we define $k$ by

$$
i(\nu)=k(\nu, T) \frac{p}{p_{0}}
$$

we have

\begin{tabular}{r|rrrrrrrr}
$\mathrm{cm}^{-1}$ & 400 & 450 & 500 & 600 & 700 & 800 & 900 & 1000 \\
\hline $\mathrm{k}_{220}$ & 2.61 & 1.43 & .96 & .54 & .35 & .23 & .18 & .14 \\
$\mathrm{k}_{300}$ & 3.50 & 1.36 & .90 & .46 & .30 & .21 & .15 & .12
\end{tabular}

The values above $500 \mathrm{~cm}^{-1}$ are very well approximated by the formula

$$
k=\frac{78,000-240 t}{(\nu-200)^{2}},
$$

which may also be extrapolated to larger values of $\nu$ than those contained in the table; $t$ is the temperature in centigrade. This formula shows that the absorption has the same character as if it were produced by a single, very strong line located at $200 \mathrm{~cm}^{-1}$. In principle a second term should be added to the one calculated above, namely the absorption caused by the band at $6 \mu$ at great distances from its center. However, if this is done the absorption turns out to be negligible compared with the one given above.

It must now be particularly emphasized that this absorption does not follow the square root law, but follows the ordinary absorption law $I=I_{0} e^{-i r}$, and the values for the absorption coefficient given in the table have to be corrected for air pressure according to formula (13). An inspection of the black-body curve shows that this type of absorption is preponderant in the spectral range covered by atmospheric radiation. The absorption coefficient rises very rapidly towards longer waves. The square root absorption treated in Section 2, however, rises still more rapidly with increasing wavelength, and between 400 and $350 \mathrm{~cm}^{-1}$ the two absorption laws give values of comparable order of magnitude. It seems a proper approximation to use the exponential absorption law alone for $\nu>375 \mathrm{~cm}^{-1}$ and to use from there on the square root law, for instance with the numerical values given in formula (9). On the short wave side, the exponential absorption law may be used up to $1250 \mathrm{~cm}^{-1}$, while from there on the square root formula (10) becomes valid. In this way the whole spectral range of the heat radiation of the atmosphere is covered.

\section{REFERENCES}

(1) W. M. Elsasser, Monthly Weather Review 65, 323 (1937), In equation (5) in this paper a factor 2 should be inserted on the right.

(2) H. M. Randall, D. M. Dennison, N. Ginsburg, and R. L. Weber, Physical Review 52, 160 (1937).

(3) F. Albrecht, Meteorolog. Zeits. 48,476 (1931).

(4) S. D. Cornell, Physical Review 51, 739 (1937).

(5) C. L. Pekeris, Astrophys. J. 79,441 (1934).

(6) W. M. Elsasser. Far infrared absorption of atmospheric water vapor. Astrophysical Jour., 87: 497-507, 1938.

(7) G. Hettner, Annalen der Physik 55, 476, (1918).

(8) F. E. Fowle, Smithsonian Mise. Coll. vol. 68, No. 6 (1917).

(9) Snithsonian Meteorological Tables, 5th ed., p. 239.

(10) W. M. Elsasser, Phys. Rev., 53, 768 (1938).

\title{
PSYCHROMETRIC CHARTS
}

\author{
By John G. Albriaht
}

\section{[Case School of Applied Science, Cleveland, Ohio]}

The accompanying psychrometric charts, constructed by the author, have been used for some time and have proved to be very helpful and convenient where extreme accuracy is not desired. They were constructed from values given in Psychrometric Tables and Relative Humidity, Centigrade Temperature, publications of the United States Weather Bureau. The values given for a barometric pressure of 29 inches were used. From these charts the relative humidity, absolute humidity and dew point can be readily found from the wet- and dry-bulb values. Chart 1 is designed for use when the temperatures are given in Fahrenheit and Chart 2 is for reference when the temperatures are given in Centigrade.

The temperatures of the dry bulb are plotted along the horizontal direction and are represented by the vertical lines, the values of which are indicated at the top. The differences between the wet- and dry-bulb thermometer temperatures are plotted along the vertical direction and are represented by the horizontal lines, the values of which are indicated at the left. Relative humidities are represented by the solid lines that slope upward to the right; their values are indicated at the right margins. Absolute humidities are represented by the broken lines that slope upward more sharply to the right; their values are indicated at the bottom of the chart.

The procedure for obtaining the relative humidity, the absolute humidity, and the dew point from wet- and drybulb thermometer readings is given in the following example. If the dry-bulb thermometer reads $90^{\circ} \mathrm{F}$. and the wet-bulb thermometer reads $80^{\circ} \mathrm{F}$., giving a 